\title{
Side-branch growth in two-dimensional dendrites. I. Experiments
}

\author{
Y. Couder, ${ }^{1}$ J. Maurer, ${ }^{1}$ R. González-Cinca, ${ }^{2}$ and A. Hernández-Machado ${ }^{3}$ \\ ${ }^{1}$ Laboratoire de Physique Statistique, Ecole Normale Supérieuere, 24 rue Lhomond, 75231 Paris Cedex 05, France \\ ${ }^{2}$ Departament de Fisica Aplicada, Universitat Politècnica de Catalunya, \\ Av. del Canal Olímpic s/n E-08860 Castelldefels (Barcelona), Spain \\ ${ }^{3}$ Departament ECM, Facultat de Fisica, Universitat de Barcelona, Diagonal 647, E-08028 Barcelona, Spain
}

(Received 9 September 2004; published 17 March 2005)

\begin{abstract}
The dynamics of growth of dendrites' side branches is investigated experimentally during the crystallization of solutions of ammonium bromide in a quasi-two-dimensional cell. Two regimes are observed. At small values of the Peclet number a self-affine fractal forms. In this regime it is known that the mean lateral front grows as $t^{0.5}$. Here the length of each individual branch is shown to grow (before being screened off) with a power-law behavior $t^{\alpha_{n}}$. The value of the exponent $\alpha_{n}\left(0.5 \leqslant \alpha_{n} \leqslant 1\right)$ is determined from the start by the strength of the initial disturbance. Coarsening then takes place, when the branches of small $\alpha_{n}$ are screened off by their neighbors. The corresponding decay of the growth of a weak branch is exponential and defined by its geometrical position relative to its dominant neighbors. These results show that the branch structure results from a deterministic growth of initially random disturbances. At large values of the Peclet number, the faster of the side branches escape and become independent dendrites. The global structure then covers a finite fraction of the two-dimensional space. The crossover between the two regimes and the spacing of these independent branches are characterized.
\end{abstract}

DOI: 10.1103/PhysRevE.71.031602

PACS number(s): 68.70.+w, 05.45.Df, 61.43.Hv, 81.10.Dn

\section{INTRODUCTION}

The growth of monocrystals in an undercooled solution gives rise to the characteristic formation of dendrites. These structures have a parabolic tip in two dimensions (a paraboloid in three dimensions) directed along one of the main crystallographic directions where the growth is fastest. Experimental, numerical, and theoretical efforts have resulted in a complete understanding of the selection mechanism [1-6] of the radius $\rho$ of the parabola in two dimensions or paraboloid in three dimensions. This type of structure is in fact an archetype of anisotropic growth in a diffusion field [7] and is also observed in other phenomena. Dendritic structures can also be obtained in Saffman-Taylor viscous fingering $[7,8]$ when a singular disturbance of the tip gives a preferred direction of growth to the viscous finger. Similar dendrites also grow in limited aggregation (DLA) in the presence of anisotropy $[9,10]$.

While the tip of the dendrite is stable, lateral protrusions are observed to form and grow. In the frame of reference moving with the dendrite tip, they form at some distance from the extremity, then grow in amplitude as they are advected away from the tip region. This is the side-branching phenomenon, which has already been widely investigated [11-23], both in three dimensions and two dimensions. As already proposed some time ago [13-16], side branching results from the selective amplification along the parabolic profile of noisy fluctuations near the tip. The initial growth of these disturbances was investigated by Dougherty and Gollub $[17,18]$. These authors showed that the very early development of the protrusions is exponential. In their experiment, the typical initial spacing of the protrusions is of the order of $5 \rho$ ( $\rho$ being the parabola radius of curvature). Both the initial amplitude and the spacing of these protrusions tend to be irregular and there is no correlation between the side branch- ing observed on each side of the dendrite. These results supported the hypothesis of the selective amplification of noise.

Correlatively, working on the dendritic fingers generated in anomalous Saffman Taylor fingering, Rabaud et al. [8] were able to apply a localized disturbance of the parabolic tip and to observe the growth and spreading of the resulting wave packet. They observed directly, in the frame of reference of the dendrite's tip, that the waves which disturb the parabolic sides are advected away from the tip with a velocity which is larger than their own. This is the characteristic feature of a class of instabilities called convective instabilities in the context of fluid dynamics where they are common in, e.g., shear flow instabilities (see Ref. [25] for a review). Their common feature is that they give rise to irregular structures because the unstable medium serves as a selective amplifier of incident noise. In such instabilities, it is possible to replace the natural noise by a periodic forcing and thus to generate a periodic structure. This was done in both dendritic viscous fingers [8] and dendrites [26,27].

The period of exponential growth of the protrusions is short, and followed by their transformation into proper side branches as they become sensitive to the crystallographic anisotropy. In the case of a cubic lattice where the main crystallographic directions of growth are (100), the branches are perpendicular to the main dendrite. As they grow the branches acquire a stable parabolic tip. The neighboring branches interact because of the nonlocal nature of the diffusion field in which they grow. Figures 1(a) and 1(b) shows two sketches of the isoconcentration lines, for slow and fast growing dendrites, respectively. The growth and interaction of the branches are expected to be different when their lengths are small compared to the local diffusion length [Fig. $1(\mathrm{a})]$ or when both length scales are of the same order of magnitude [Fig. 1(b)]. 


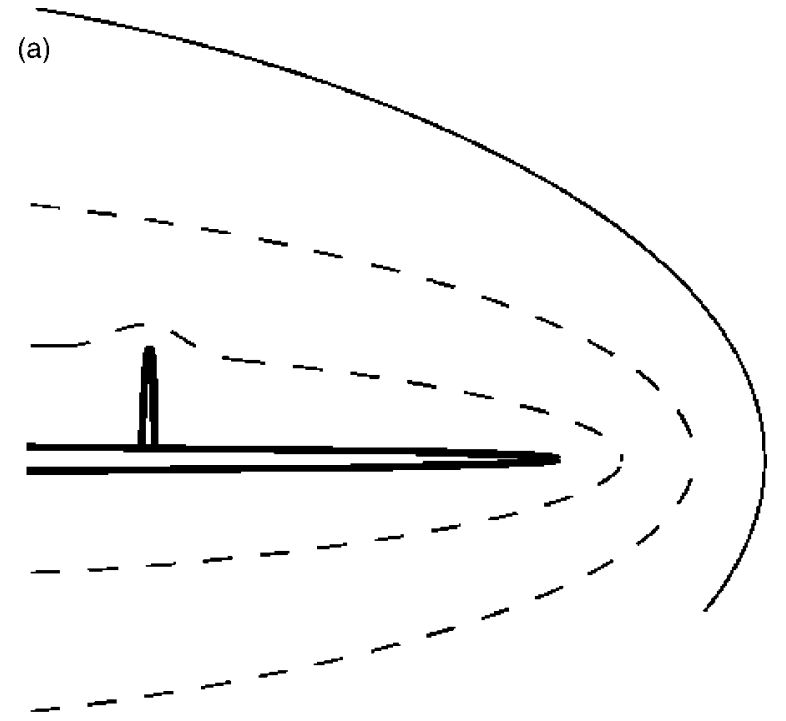

(b)

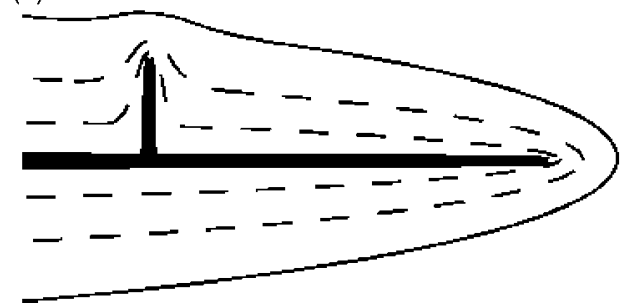

FIG. 1. (a), (b) Sketches of two dendrites growing at small and large velocity respectively and of the isoconcentration lines in their vicinity.

A theoretical incentive for the study of the growth of slow dendrites on a large scale comes from results obtained on anisotropic DLA, which showed the formation of a selfaffine fractal structure $[9,10]$. In the context of crystal growth a fractal regime is only observed in the limit of very longrange interaction and thus very large diffusion length. This was investigated in the quasi-two-dimensional situation by Couder et al. [19], in experiments performed in the crystallization of ammonium bromide diluted in water and contained in a quasi-two-dimensional cell. Ahead of a crystallization front growing at a velocity $U$ the impurity diffusive field is characterized by its length scale $l_{\mathrm{D}}=2 D / U$ where $D$ is the diffusion constant. At weak under-cooling, the growth is slow and $l_{\mathrm{D}}$ is so large that the distances between all branches are small in comparison. In these conditions an approximation of a Laplacian growth is obtained and the side branches are not free: they remain prisoners of the lateral front of the main dendrite. Their velocity is not constant and they keep interacting, screening off the slow branches, so that the structure continues coarsening. This leads to the buildup of a self-affine fractal structure. The fractal dimension of such a dendrite was measured [19], far from the tip, by a box counting method and found to be $d_{\mathrm{f}}=1.58 \pm 0.03$ in agreement with the dimension $\left(d_{\mathrm{f}}=1.57\right)$ found [10] for fourfold anisotropic DLA.

In the same limit, the area occupied by the dendrite in two dimensions was also investigated [19]. In spite of the fluc- tuations due to the side-branching instability, this area is the same (on the average) as that of a smooth parabolic shape. The parameter of this average parabola is the same as that of the stable dendrite. This means that the mean fractal object occupies the same area that the dendrite would have covered if it had remained a stable parabola with tip radius $\rho$. This parabolic dependence of the unstable dendrite could be expected from Ivantsov's [1] qualitative argument: the tip of the dendrite moves at constant velocity so that its position is proportional to time $t$; the growth of the lateral sides results from a diffusive process and their position moves as $t^{0.5}$.

In three dimensions the situation is slightly more complicated [20-22]. The dendrite has the shape of a paraboloid only near the tip but the axisymmetry around the dendrite axis breaks down even before the lateral destabilization. First there is formation of four ridges which then destabilize to form side branches. These grow in the main crystallographic directions perpendicular to the dendrite's direction. The cross section of the dendrite thus develops in a four armed petal shaped structure. If $x^{\prime}$ is their distance to the tip, the theoretical prediction is that the mean length of these arms [21] grows as $\left|x^{\prime}\right|^{3 / 5}$ (i.e., as $t^{3 / 5}$ ) while their width in the orthoradial direction grows as $\left|x^{\prime}\right|^{1 / 5}$. The growth in a paraboloid mean shape is only recovered if an averaging is done over all the radial directions around the axis of the dendrite.

In the present paper we will perform experiments aimed at characterizing the late evolution of the side branches in an approximately two-dimensional situation.

We will first study the regime of growth at very small Peclet numbers. The work will be focused on the characterization of the coarsening phenomenon responsible for the buildup of ever larger branches. This will shed light on how the growth of the average parabolic front can be sustained by an ever-decreasing number of branches. Some preliminary results about it were published in Ref. [24].

We will then turn to the specific effects observed for larger velocities and smaller $l_{\mathrm{D}}$. The same initial coarsening leads to a greater spacing of the growing side branches. But when this spacing becomes of the order of the local thickness of the mean diffusion front, the side branches have the possibility of escaping and form independent dendrites.

Finally this first paper (Part I) is exclusively experimental. Part II is devoted to a phase field numerical computation of this growth of the dendrites lateral branches.

\section{EXPERIMENTAL SETUP}

We grow ammonium bromide crystals from supersaturated aqueous solution in conditions similar to those previously described $[19,28]$. The solution, of concentration $c_{o}$, is initially enclosed in a thin cell formed of two glass plates of radius $50 \mathrm{~mm}$. The distance between the plates is fixed by a mylar spacer of thickness $e=20 \mu \mathrm{m}$. The cell is entirely immersed in a circulation of water thermally regulated with an accuracy of $20 \mathrm{mK}$. A few runs are first performed at large undercooling so as to obtain several crystals in the cell. The cell is then warmed up so as to melt these crystals almost completely, leaving only a few germs. The desired undercooling is then imposed and the growth of the dendrites can 
be observed. The experiment is only recorded when one isolated, well oriented germ (with two [100] axis in the plane of the cell) has been obtained. We then wait until a long dendrite is obtained, far away from the boundaries and from the other main dendrites grown from the same germ.

As the thermal diffusivity is three orders of magnitude larger than the mass diffusivity, the system is limited by mass diffusion for which $D=2 \times 10^{-9} \mathrm{~m}^{2} / \mathrm{s}$. Several different solutions were used for these experiments with concentrations corresponding to several equilibrium temperature $T_{\mathrm{E}}$. Solutions with $T_{\mathrm{E}}=35^{\circ} \mathrm{C}$ were chosen for the cases where a small undercooling was to be applied, leading to slowly growing dendrites. Fast dendrites were grown in solutions with an equilibrium temperature. $T_{\mathrm{E}}=50{ }^{\circ} \mathrm{C}$ where undercoolings as large as $35^{\circ} \mathrm{C}$ could be chosen. Altogether, dendrites growing in a very large range of velocities $3-90 \mu \mathrm{m} / \mathrm{s}$ were thus obtained.

The dendrites were observed with an inverted microscope which allowed magnifications up to 480 . The growth was recorded with a video camera and digitized with a resolution 768 by 512 pixels. We used four different magnifications corresponding respectively to resolutions $0.36,1.19,2.97$, and $4.76 \mu /$ pixel. Since we wanted to record the growth of individual branches until they reached large amplitudes, we generally used the magnification corresponding to $2.97 \mu \mathrm{m} /$ pixel so that the early growth of the side branching was poorly resolved. For this reason, in the case of the dendrites of low velocities, before recording the branch growth, we briefly recorded the growth of the tip with a large magnification so as to measure its shape. The results, concerning the relation between the selected radius of curvature and the velocity, were in good agreement with the values previously reported [28].

Finally we can note that in spite of its small thickness $(e=20 \mu \mathrm{m})$, the cell does not impose a strict two dimensionality to the growth. The tip, having a radius smaller than $d$, remains a three-dimensional paraboloid. However, the limited thickness prevents the growth of branches in the direction perpendicular to the cell's plane. Furthermore, for the slow dendrites, the cell thickness is always small compared to the distance of the competing branches. The diffusion field in which these branches grow can thus be considered as two dimensional. The fact that most of our results are recovered in strictly two-dimensional simulations (see Part II) is confirmation of the validity of the two-dimensional (2D) aproximation for the investigation of the side branches' growth.

\section{EXPERIMENTAL RESULTS}

\section{A. The self-affine growth, phenomenological characterization of coarsening \\ 1. Global structure}

We will first investigate the regime of slow growth at weak undercoolings. A well oriented initial seed in a flat cell gives rise to four arms. The diffusion length being large, these arms interact with each other so that each of them grows in a petal shape [29]. Only the front part of each petal, can be considered parabolic. For this reason we always waited long enough for the dendrite to have grown far away from the germ. In the following, the axes of the laboratory frame of reference are chosen so that the dendrite grows along the $O_{x}$ axis and its branches along $O_{y}$. Taking the origin $O^{\prime}$ at the dendrite's tip $O_{x^{\prime}}^{\prime}, O^{\prime}{ }_{y^{\prime}}$ will be the frame of reference moving with it.

The results we will first report in detail were obtained on a dendrite which grew with a velocity $V=3.77 \mu \mathrm{m} / \mathrm{s}$. Using a strong magnification we measured by image processing [28] the shape of the tip parabola and deduced a tip radius $\rho=1.4 \pm 0.1 \mu \mathrm{m}$. This value corresponds to a value $\rho^{2} V=k$ $=7.5 \mu \mathrm{m}^{3} / \mathrm{s}$ which is in good agreement with the value found for crystals of $\mathrm{NH}_{4} \mathrm{Br}$ grown in a solution with $T_{\mathrm{E}}$ $=35^{\circ} \mathrm{C}$, as reported by Maurer et al. [28].

We then turn our interest to the large scale structure of the dendrite. The system is limited by mass diffusion for which the diffusion constant is $D=210^{-9} \mathrm{~m}^{2} / \mathrm{s}$, so that for this dendrite the Peclet number $(\mathrm{Pe}=\rho V / 2 D)$ is $\mathrm{Pe} \sim 10^{-3}$. At such a low velocity of growth the diffusion length is large and a self-affine regime of growth is observed in a large range of length scales. As in our previous work (Couder et al. [19]) the half area covered by the structure (on the $y^{\prime}>0$ side) grows (on the average) with the distance to the tip as

$$
S\left(x^{\prime}\right)=\left(\frac{8 \rho}{9}\right)^{1 / 2} x^{\prime 3 / 2},
$$

where $x^{\prime}$ is the distance to the tip. This means that the selfaffine dendrite has a mean lateral front. The same front that would have existed if the dendrite had not undergone a lateral destabilization and had remained parabolic. It is thus located at

$$
y_{d}^{\prime}=\left(2 \rho x^{\prime}\right)^{1 / 2} .
$$

In the laboratory frame of reference, at a large distance $x^{\prime}$ from the tip, the velocity normal to this front is thus on the average

$$
\bar{U}_{N}=(\rho / 2)^{1 / 2} V\left(x^{\prime}\right)^{-1 / 2} .
$$

Taking into account the selection of the tip radius by which $\rho^{2} V$ is a constant $k$ and the normal velocity becomes

$$
\bar{U}_{N} \approx 2^{-1 / 2} k^{1 / 4} V^{1 / 4} t^{-1 / 2}
$$

The decrease of the normal velocity along the mean parabolic profile induces an increase of the diffusion length. In the tip region it is $l_{D}^{T}=2 D / V$. But along the sides the diffusion length, $l_{D}^{S}$ grows with the distance to the tip as

$$
l_{D}^{S}=\left(\frac{2 D}{V} \sqrt{\frac{2}{\rho}}\right) x^{\prime 0.5}
$$

in a given region of the laboratory frame of reference, the diffusion length grows as the square root of time,

$$
l_{D}^{S} \approx 2 \sqrt{2} D k^{-1 / 4} V^{-1 / 4} t^{1 / 2} .
$$

In the case of the dendrite growing at $V=3.77 \mu \mathrm{m} / \mathrm{s}$, the diffusion length at the tip is $l_{D}^{T}=0.53 \mathrm{~mm}$. In Fig. 2 going from (b) to (d) the normal velocity of the mean front decreases from $\sim 0.1$ to $\sim 0.06 \mu \mathrm{m} / \mathrm{s}$. Correlatively, the diffu- 


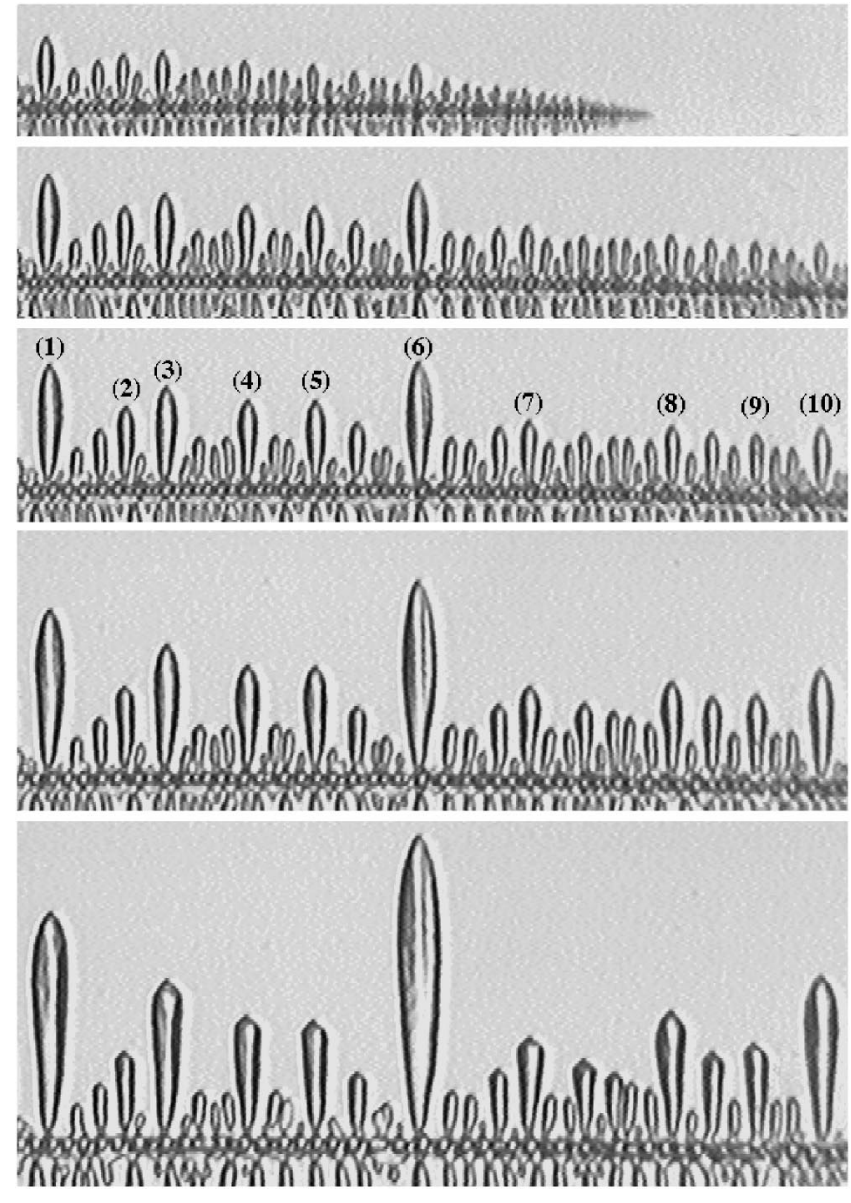

FIG. 2. Five photographs taken respectively at a time $t_{o}$ and $t_{o}$ $+300 \mathrm{~s}, t_{o}+660 \mathrm{~s}, t_{o}+1260 \mathrm{~s}, t_{o}+3180 \mathrm{~s}$, of one side of a dendrite growing at low velocity $V=3.77 \mu \mathrm{m} / \mathrm{s}$. The length of the region shown here is of the order of $L=2.2 \mathrm{~mm}$. The labels (1) $\cdots(10)$ show the branches which have been investigated.

sion length grows from $l_{D}^{S} \sim 2 \mathrm{~mm}$ to $\sim 3 \mathrm{~cm}$. These values are always very large as compared to the size of the branches and to their spacing. The cell thickness is small as compared to $l_{D}^{S}$, so that the diffusion field can be considered as two dimensional.

\section{The growth of the dominant branches}

The dendrite is observed with a microscope and a video camera which records a fixed field that the dendrite crosses during its growth. Figure 2 shows five images of such a recording concerning $2.2 \mathrm{~mm}$ of the length of the dendrite. Note that this region was more than $1 \mathrm{~cm}$ away from the initial seed so that the influence of the other dendrites grown from the same seed was negligible. In this case the growth was observed during a period of an hour and a half. Figure 2(a) is typical of the first frames where the tip region of the dendrite is observed. The distribution of branches resulting from the primary instability form an irregular array with branches having an initial average spacing of approximately $5 \rho$ and various amplitudes. The four others show the typical evolution of the side branches. The process of coarsening is clearly visible: fewer and fewer branches continue growing as time elapses. The branches which stop growing remain as fossil structures. Much later, however, it can be noted that the tip of these branches becomes facetted, before eventually melting. The sequence shown in Fig. 2 exhibits another obvious feature. The largest branches were already locally dominant at their formation. In other terms, the dominance of a branch appears to be entirely determined from the start. The following measurements set this observation on a quantitative basis.

On an image such as Fig. 2(c) we use a home-made image processing which detects the profile of the dendrite, then its protrusions in the $O_{y}$ direction. Each of these protrusions is ascribed a number $n$. It is easy to follow a branch on the successive images because its abscissa $x_{n}$ is practically constant. The origin of the time $t_{n}$ used for a given branch $n$ is the time when the tip of the main dendrite has passed at the abscissa $x_{n}$. We measure the position $y_{n}\left(t_{n}\right)$ of the tip of each branch as a function of its own time.

Figure 3(a) shows the plot $y_{n}\left(t_{n}\right)$ of the growing length of five of the branches of the dendrite shown in Fig. 2. They all have a velocity which decreases as a function of time. This means that none of the lateral branches becomes a free dendrite. All branches remain prisoner of the diffusion front of the main dendrite. In a loose sense, the lateral branches can thus be considered as dendrites resulting from the instability of a directional growth in the mean diffusion field imposed by the main dendrite. As time elapses the front slows down and this diffusive field thickens: correlatively there is coarsening and the wavelength separating active branches keeps increasing.

A remarkable result is that the hierarchy of size of the branches is fixed initially at the formation of the lateral branches. A better characterization is obtained in a log-log plot [Fig. 3(b)] where each branch is observed to be fitted by a power law growth of the type

$$
y_{n}(t)=y_{n}^{o} t_{n}^{\alpha_{n}} .
$$

The velocities thus all decrease with time as $V_{n} \propto t^{\alpha_{n}-1}$. The exponent $\alpha_{n}$ is constant for a given branch but depends on how dominant the branch is at the start, i.e., how strong the initial disturbance was. The values of the $\alpha_{n}$ for the branches of Fig. 2 are given in Table I. For this dendrite, they range from 0.51 to 0.64 . We can note that this power law behavior is only observed in a finite range of values of $t$. At large times the velocity of all observed branches decreases due to their screening off by faster branches. Even branch 6, which is the fastest, is observed to slow down at times larger than $2000 \mathrm{~s}$ as it begins to be screened off by a larger branch located outside the observed region. This screening effect is analyzed in Sec. III A 3.

These results suggest that the stronger the initial disturbance, the larger the growth exponents. For this reason it is interesting to observe situations in which a dendrite is submitted accidentally to a strong localized disturbance. Figure 4 shows such an event where a dendrite of velocity $7.7 \mu \mathrm{m} / \mathrm{s}$ collided with a speck of dust present in the cell. The violent disturbance of the tip leads to a symmetrical destabilization of the front which already has a finite amplitude very close to the tip [Fig. 4(a)]. As a result, two symmetrical branches 

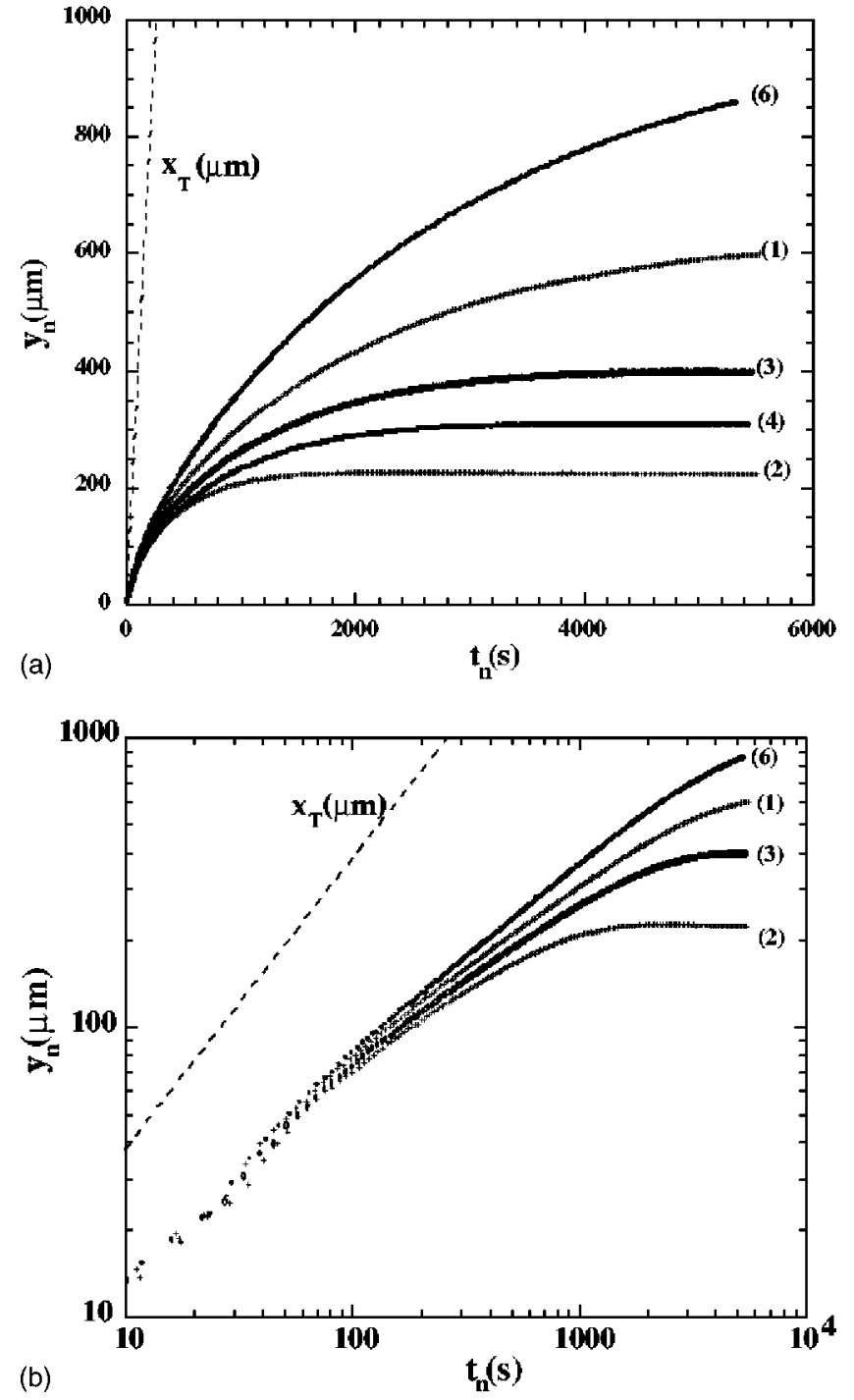

FIG. 3. (a) The lengths $y_{n}\left(t_{n}\right)$ of the lateral branches (1), (2), (3), (4), (6), as a function of time (in seconds). Branches 2 and 4 reach limit lengths $y_{2}^{\text {Max }}$ and $y_{4}^{M a x}$, respectively. The dotted line shows the linear motion $x_{T}(t)$ of the tip of the dendrite on the same scale. (b) Logarithmic plot for branches (1), (2), (3), and (6). The best fits for the power law regions correspond to $\alpha_{1}=0.58, \alpha_{2}=0.51, \alpha_{3}=0.54$, $\alpha_{6}=0.64$, respectively.

form, which grow faster than usual. Their initial growth velocity is close to being $V / 2$, half of the velocity of the main dendrite, (see below Sec. III B 2). The logarithmic plot (Fig. 5) shows that these branches also have a power law growth of the type given by Eq. (7) but with an unusually large value $\alpha=0.80$. Such branches are naturally dominant over their neighbors. In this range of Peclet numbers and in observed regions as large as $1 \mathrm{~cm}$ they are observed to slow down $(\alpha<1)$ and thus they still remain prisoner of the mean lateral front.

\section{The slower branches' screening off}

The second process is screening off. As time passes, the slower branches stop growing altogether. This is observed in
TABLE I. Growth exponent $\alpha_{n}$ and screening parameters $Y_{o}$ and $S_{o}$ for branches $1-8$.

\begin{tabular}{cccc}
\hline \hline Branch & $\alpha_{n}$ & $Y_{o}$ & $S_{o}$ \\
\hline 1 & $0.58 \pm 0.01$ & & \\
2 & $0.51 \pm 0.01$ & 0.7 & 0.5 \\
3 & $0.54 \pm 0.01$ & 0.75 & 0.45 \\
4 & $0.53 \pm 0.01$ & 0.77 & 0.4 \\
5 & $0.54 \pm 0.01$ & 0.73 & 0.45 \\
6 & $0.64 \pm 0.01$ & & \\
7 & $0.52 \pm 0.01$ & 1.0 & 0.46 \\
8 & $0.57 \pm 0.01$ & 0.9 & 0.42 \\
\hline \hline
\end{tabular}

Fig. 3 where the branches (e.g., 2 and 4) reach asymptotic lengths, $y_{2}^{\operatorname{Max}}$ and $y_{4}^{\operatorname{Max}}$, respectively. These branches have stopped growing because of the screening effect due to faster growing branches. This effect is dominated by the geometrical positions of neighboring branches as can be observed in Fig. 2. Neglecting the curvature of the parabola near the tip, the relative position of these branches can be sketched [Fig. 6(a)] as uneven branches growing from a linear base. On this sketch we draw two straight lines from the extremity of each branch and tangent to the most protruding neighboring structures. Whenever these neighbors are larger, they have larger $\alpha_{n}$ 's so that the difference in size will increase with time. The velocity of the small branch then decreases until it finally stops completely. This process repeats itself, leading to a hierarchy of screening-off processes proceeding from the nearest to the furthest. On Fig. 6(a) branch $n$ will be screened
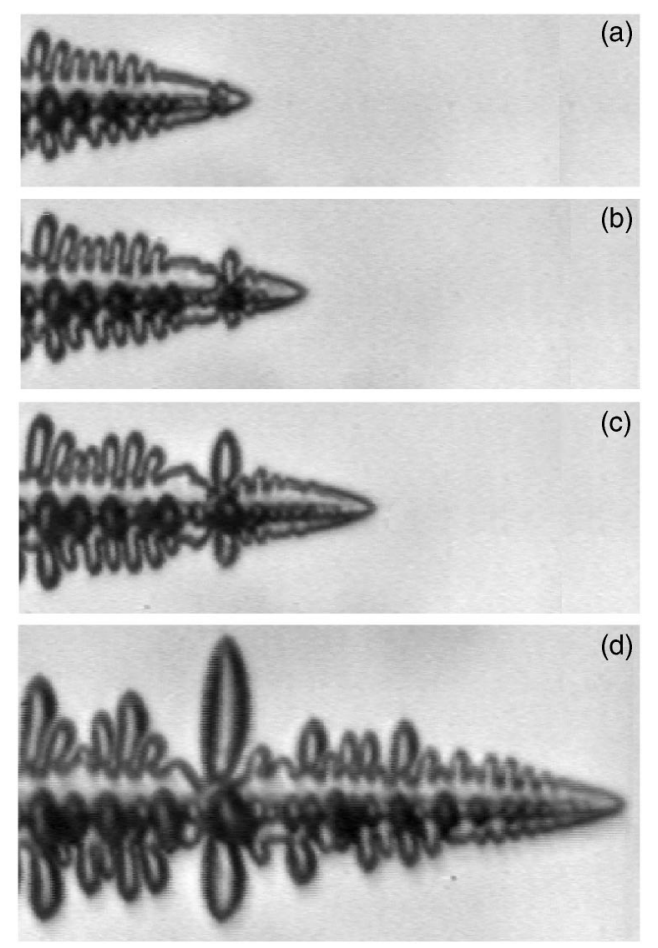

FIG. 4. Four photographs taken, respectively, 1, 3, 5.4, and $15.1 \mathrm{~s}$, after the collision of a dendrite growing at velocity $V$ $=7.7 \mu \mathrm{m} / \mathrm{s}$ with a finite disturbance. 


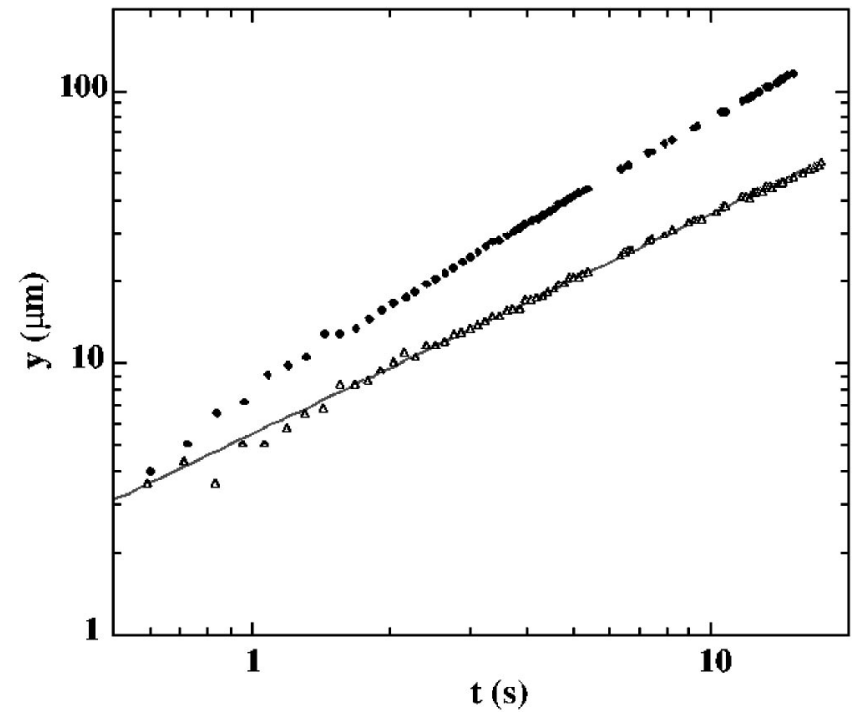

FIG. 5. Logarithmic plot of the position of the tip of the disturbed dendrite of Fig. 4 and of the length of its main lateral branch as a function of time (in seconds). This branch grows as $t^{\alpha_{p}}$ with $\alpha_{p}=0.80$.

off by $p$ and $q$, then branch $p$ will be screened off by $q$ and $r$ and later $q$ will stop because of the influence of $r$ and $s$. The process repeats itself on larger and larger scales.

If $y_{n}^{\text {Max }}$ is the maximum length reached by branch $n$ we define the nondimensional quantity $Y_{n}$ as

$$
Y_{n}=\frac{y_{n}^{\operatorname{Max}}-y_{n}}{y_{n}^{\operatorname{Max}}} .
$$

The geometrical situation of the branch $n$ between $p$ and $q$ is characterized by the screening angles $\theta_{n}^{p}$ and $\theta_{n}^{q}$ as sketched in Fig. 6(b). It turns out that the dynamically relevant parameter is

(a)

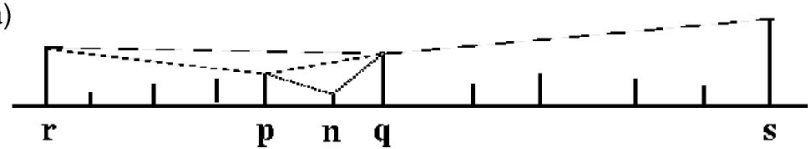

(b)

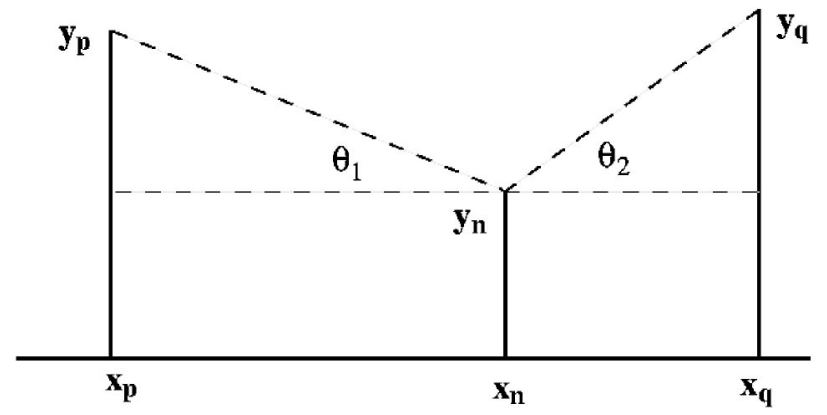

FIG. 6. (a) Sketch of the relative positions of branches resulting from the primary instability. (b) Definition of the screening angles $\theta_{n}^{p}$ and $\theta_{n}^{q}$ when branch $n$ is screened off by branches $p$ and $q$.
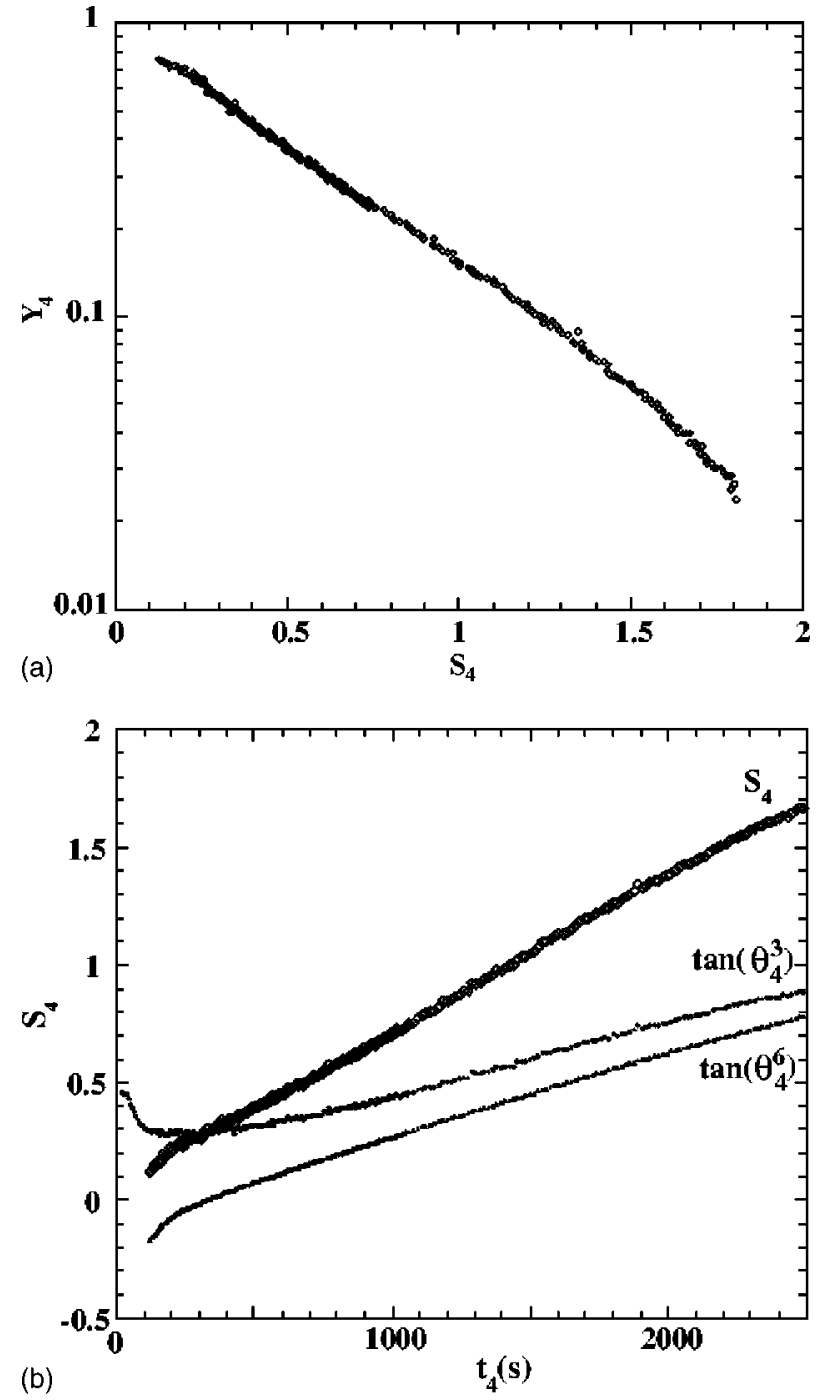

FIG. 7. (a) Semilogarithmic plot of $Y_{4}$ as a function of $S_{4}$, showing the range of logarithmic decay of the growth of branch 4 of Fig. 2 when screened off by branches 3 and 6 . (b) Linear plot of $\tan \theta_{4}^{3}$ and $\tan \theta_{4}^{6}$ and of their sum $S_{4}$ as a function of $t_{4}$.

$$
S_{n}=\tan \theta_{n}^{p}+\tan \theta_{n}^{q}
$$

with $\tan \theta_{n}^{p}=\left(y_{p}-y_{n}\right) /\left(x_{p}-x_{n}\right)$ and $\tan \theta_{n}^{q}=\left(y_{q}-y_{n}\right) /\left(x_{q}-x_{n}\right)$. The time evolution of $S_{n}$ can be computed from the measured $y_{n}\left(t_{n}\right), y_{p}\left(t_{p}\right), y_{q}\left(t_{q}\right)$. Figure 7(a) is a semilogarithmic plot of $Y_{n}(t)$ as a function of $S_{n}(t)$ for branch 4. An exponential decay is clearly observed showing that $Y_{n}$ and $S_{n}$ satisfy

$$
Y_{n}=Y_{0} \exp \left(-S_{n} / S_{0}\right)
$$

The two parameters $Y_{0}$ and $S_{0}$ are obtained from the best fits of the linear part of the semilog plots [Fig. 7(a)]. The values found for various branches are approximately constant and given in Table I. It can be noted that they depend neither on the scale of the process nor on the symmetry or asymmetry of the positions of branches $p$ and $q$ with regard to branch $n$. The mean value of $S_{0}$ is 0.44 . The values of $Y_{0}$ are more scattered. When the screening-off process of branch $n$ begins early we find values of $Y_{0} \sim 1$. This value is found 
when branch $n$ is mainly screened by a previous branch $p$. In the opposite situation where the main screening comes from a branch $q$, grown after $n$, we obtain $Y_{0} \sim 0.75$. From these values we can deduce that in a symmetrical situation, branch $n$ will reach an amplitude $0.99 y_{n}^{\operatorname{Max}}$ for $\theta_{n}^{q}=\theta_{n}^{q}=45.4^{\circ}\left(Y_{0}\right.$ $\sim 1)$ or for $\theta_{n}^{q}=\theta_{n}^{q}=43.5^{\circ}\left(Y_{0} \sim 0.75\right)$.

We have thus defined the screening-off process in purely geometrical terms. Can this lead to a temporal description of the growth of a given branch? We find empirically that the time evolution of the screening factor is linear [see Fig. 7(b)] so that $S$ can be written

$$
S=a+b t_{n}
$$

as a result combining relations (8) and (9) the time evolution of the branch length can be written

$$
y_{n}=y_{n}^{\operatorname{Max}}\left[1-\lambda \exp \left(-\mu t_{n}\right)\right]
$$

$$
\text { with } \lambda=Y_{0} \exp \left(-a / S_{0}\right) \text { and } \mu=b / S_{0} .
$$

The growth of an infinite array of parallel needles in a Laplacian field was investigated by M. Adda Bedia [30]. In his lattice shorter and larger needles alternate. The smaller needles are then observed to undergo an exponential decay. There are differences between this theoretical situation and the experimental one. For instance the lattice of parallel needles is infinite, and there is no central base from which the needles grow. However, the screening effect is of the same nature as observed experimentally.

\section{B. Growth at large velocities}

\section{Global structure}

The global structures of slow and fast 2D dendrites are very different. As discussed above, the slow dendrites are self-affine fractals which retain a global parabolic mean shape. The velocities of their branches are always small compared to that of the main dendritic tip. In contrast, the fast dendrites cover a finite fraction of the 2D space and have a wedge shaped envelope as a result of the velocity of the branches which equals that of the main tip. The investigation of the growth of individual branches sheds some light on the origin of the crossover between these two regimes of growth. Two main features are observed which will be described successively. Near the tip there is a quantitative change in the values of the growth exponents. Far from the tip, there is a qualitative change as some branches escape from the diffusion field of the main dendrite.

\section{The initial behavior of the fast branches}

In the range of velocities $20<V<50 \mu \mathrm{m} / \mathrm{s}$, as the diffusion length diminishes, different processes appear, both near the tip and away from it. In all this range of velocities the dendrite is still observed to have a classical parabolic extremity and the same mode of initial destabilization. The above-described processes of competition between branches are still observed. However, compared to the previous experiments the rate of growth of the lateral instability is now larger and the destabilization of the parabolic front occurs

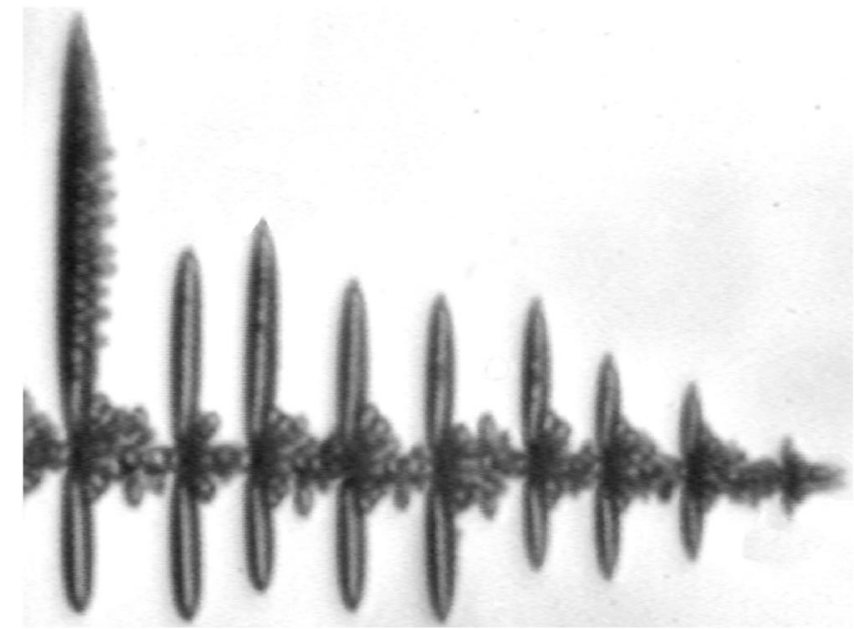

FIG. 8. Photograph of the tip of a fast dendrite in the regime of tip oscillations.

nearer to the tip. Correlatively there is a shift of the values of the growth exponents $\alpha_{n}$ resulting from the normal fluctuations. They are found to range from 0.6 to 0.9 for a dendrite of velocity $20 \mu \mathrm{m} / \mathrm{s}$ and from 0.8 to 1 for a dendrite of velocity $50 \mu \mathrm{m} / \mathrm{s}$. As previously, the value of an $\alpha_{n}$ depends on the amplitude of the initial disturbance of the parabolic front. For $V \geqslant 50 \mu \mathrm{m} / \mathrm{s}$ large amplitude disturbances occur more frequently and generate branches which grow with $\alpha$ $=1$ and thus a constant velocity. A remarkable result is that in those cases the branch velocity is initially half that of the dendrite (as will be seen below in Fig. 10). This is in agreement with a recent finding by Hakim [31] in a model system. Working on linear needles growing in a Laplacian field, he considered a situation where, on a semi-infinite needle, two lateral branches grow at a right angle. He finds analytically a specific solution in the shape of a cross where the velocity of the two branches is half that of the main needle.

In the range of velocities $50<V<100 \mu \mathrm{m} / \mathrm{s}$ the tip itself appears strongly disturbed by instabilities which generate frequent and large lateral disturbances. In this instability the tip suddenly slows down and splits asymetrically, generating two secondary tips of unequal length. Unlike what is observed at larger velocities, this tip splitting fails to produce two independent structures. The larger of the two tips accelerates while the other stops. This leads to the formation of a new single tip, which in turn will become unstable by a new tip splitting. The whole structure thus undergoes relaxation oscillations. It is not known if this is an intrinsic destabilization mode, or if this instability is generated by an interaction with the cell's walls. A quantitative investigation of the tip structure in this range of velocities would have required working with a fast camera and was not the aim of the present paper. It is worth mentioning, however, because of its implication on the behavior of lateral branches on a long time range. Each tip splitting is a strong disturbance where two symmetrical side branches of large amplitude are generated (Fig. 8). Finally, at very large velocities the tip of the dendrite can also undergo a complete tip splitting. As shown in Fig. 9, this gives rise to two dendrites which will continue growing alongside each other. 


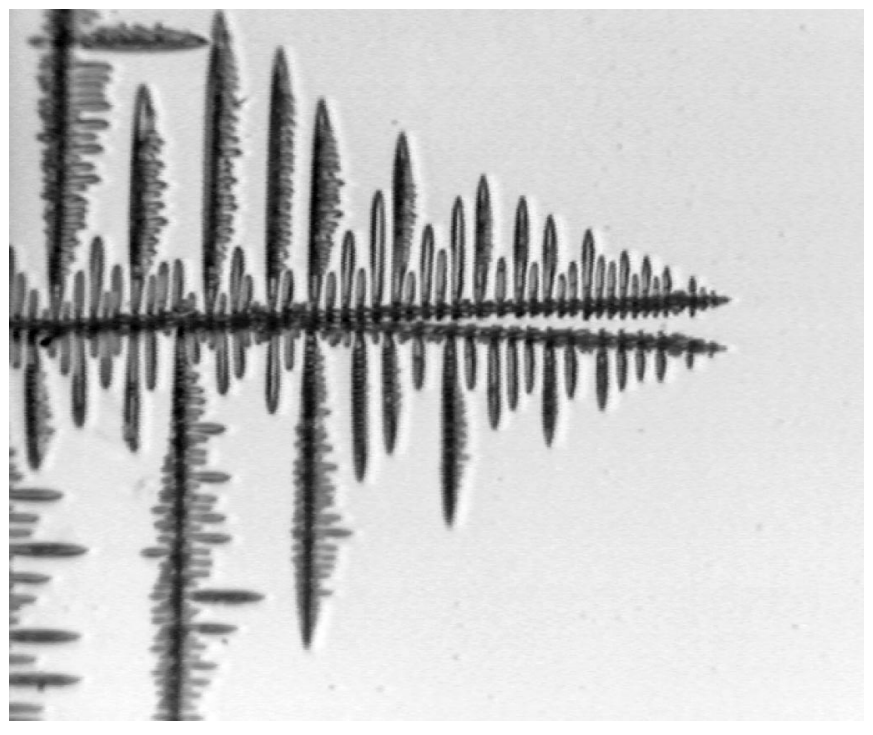

FIG. 9. Photograph of a dendrite of velocity $V=51 \mu \mathrm{m} / \mathrm{s}$ after its tip has split.

\section{The escape of branches}

We can now turn to the evolution of branches, as observed on a long time scale, using a weaker magnification of the microscope.

For dendrites with $20<V<50 \mu \mathrm{m} / \mathrm{s}$, the branches usually have the same evolution as described in Sec. III A: there is coarsening and fewer and fewer branches continue growing as time elapses. In the cases where the dendrite has undergone a finite disturbance something different occurs. At some distance away from the tip the resulting branch is observed to accelerate (Fig. 10, inset), before again reaching a constant velocity. Three successive regimes of growth are clearly identified on the plot of the length of the dominant

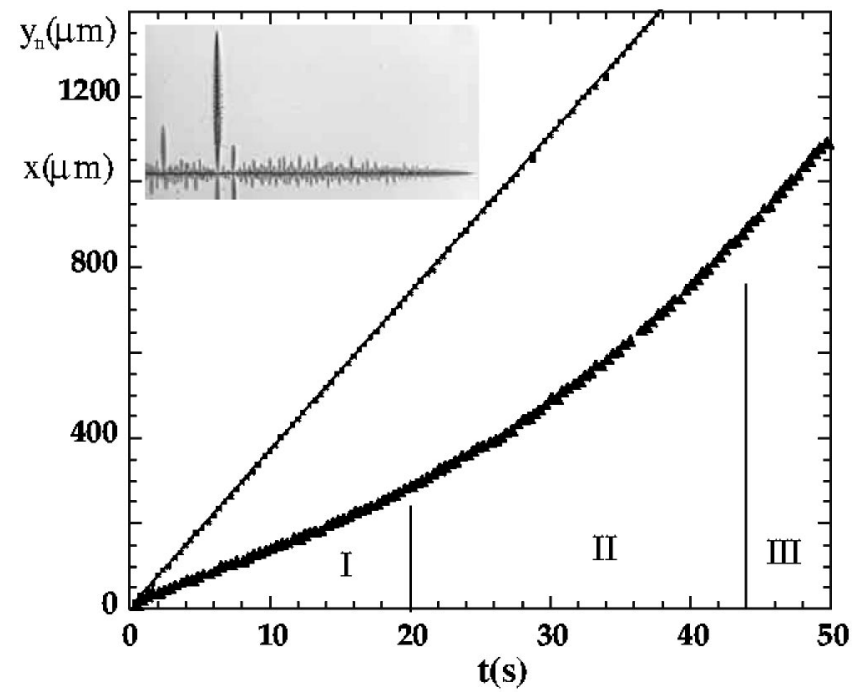

FIG. 10. Linear plot of the position of the tip of a dendrite grown at $V=39.9 \mu \mathrm{m} / \mathrm{s}$ and of its main lateral branch as a function of time. The three regimes of growth are labeled I, II, and III. The time of escape $\tau$ is the limit separating II from III. Inset: photograph of this dendrite. branch as a function of time (Fig. 10). In the initial region (I) the branch is prisoner of the main dendrite and grows with a power law of time (on the example shown here $\alpha=1$ ). Then it accelerates (region II). Ultimately, in region III, the velocity becomes constant and equal to the velocity $V$ of the main dendrite. The branch has then escaped the main dendrite's diffusion front, to become an independent dendrite. For dendrites with $V<40 \mu \mathrm{m} / \mathrm{s}$, this escape was only observed to occur for those fast branches which resulted from an anomalous strong disturbance of the tip. If a dendrite is grown in the same conditions as those of Fig. 10, but without undergoing any finite disturbance, no branch escapes in the limited duration of our experiment.

Four successive images of a faster dendrite ( $V$ $=51 \mu \mathrm{m} / \mathrm{s}$ ) are shown in Fig. 11. In the tip region the structure is similar to that discussed above, and the branches are still disturbances of a dendrite which remains parabolic on the average. At a certain distance from the tip some branches are repeatedly observed to accelerate until they reach the velocity $V$ of the main dendrite. The most obvious feature of the side of the global structure of a fast dendrite is that it is formed of independent parallel branches, all growing at the same velocity (Figs. 11 and 12). On a given dendrite, the branches which escape reach the same velocity after approximately the same delay $\tau$, so that the global structure acquires a wedge shape. This organization is obvious on a photograph (Fig. 12) of a very fast dendrite $(V=87 \mu \mathrm{m} / \mathrm{s})$ at a weak magnification. Two straight lines at $45^{\circ}$ from the direction of the initial dendrite form the envelope of the branches. The structure, though complex, is no longer a self-affine fractal; inside the wedge the pattern covers a finite fraction of the $2 \mathrm{D}$ space. The tip of the main dendrite protrudes slightly at the vertex of the wedge. This is related to the delay necessary for the branches to free themselves. At large velocities, branches growing with $\alpha \sim 1$ become frequent as the loss of stability of the tip creates large initial disturbances, and the diffusive field becomes thinner. As a result the initial region of growth (region I in Fig. 10) becomes shorter and the fit by a power law becomes less and less accurate. Some branches are observed to have a velocity increasing with time very shortly after their formation. It is not clear if they have a growth exponent $\alpha>1$ or if this is the effect of the crossover regime of region II.

Two typical lengths can be measured. The first is $l_{\mathrm{E}}$ the distance from the tip at which the branches become free. This length is $l_{\mathrm{E}}=\tau V$ where $\tau$ is the typical escape time of the branches. This time $\tau$ is measured with a rather good accuracy on plots of the type of Fig. 10, where it is the limit between regions II and III. On various dendrites obtained at different undercoolings the evolution of this time with the velocity was measured. As shown in Fig. 13(a), $\tau$ diverges at low velocities. The second typical length is the distance between free branches. Since they are not evenly spaced, we measured the distances $\lambda$ of as many free branches as possible and deduced an average value $\bar{\lambda}$. We then repeated the measurements on dendrites grown at various velocities. In the low range of velocities the free branches are scarce so that the average values are not precise. For a given dendrite the two lengths $l_{\mathrm{E}}=\tau V$ and $\bar{\lambda}$ have similar values. 

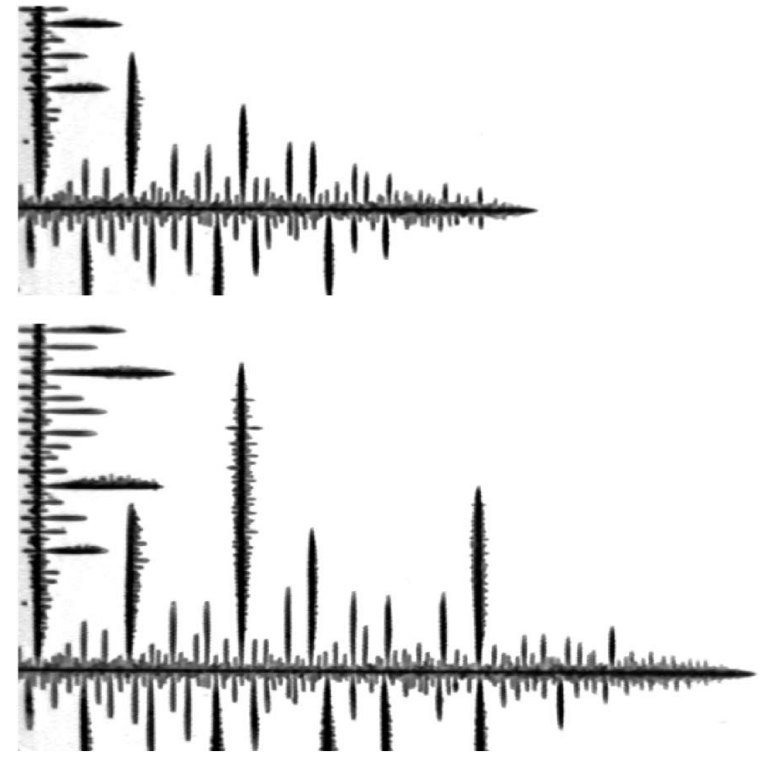

(b)
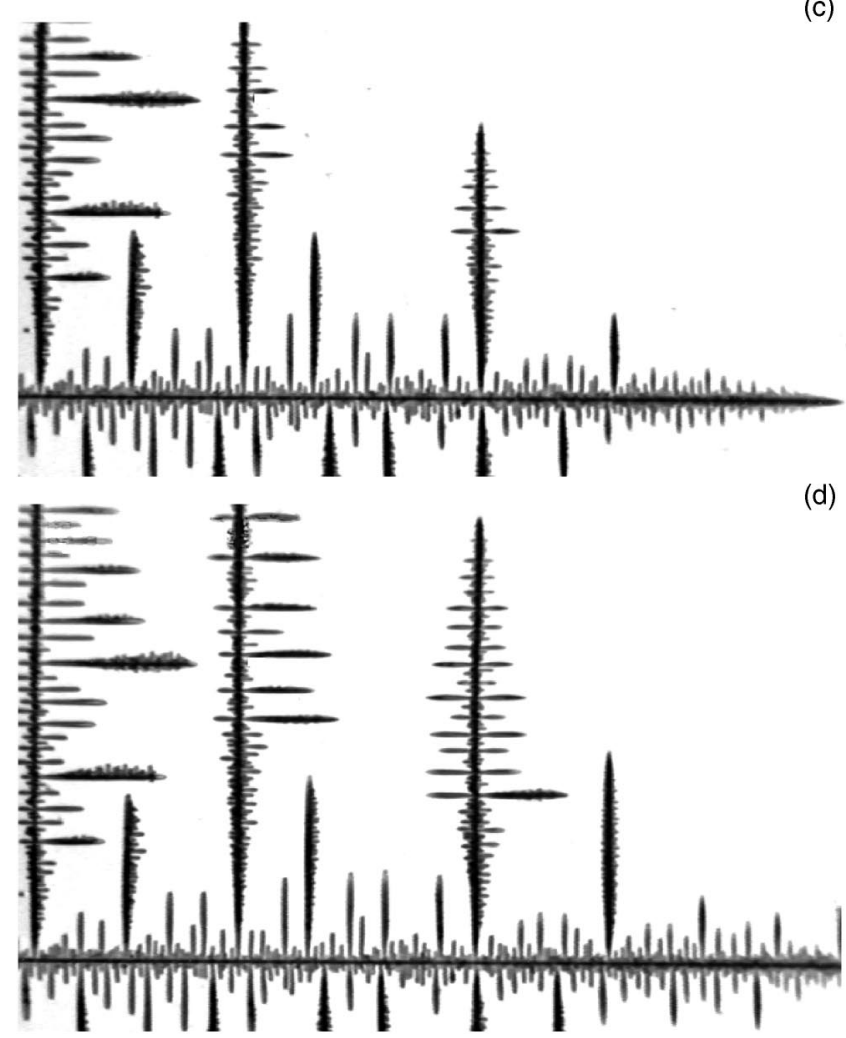

FIG. 11. Four photographs taken respectively at a time $t_{o}$ and $t_{o}+9.2 \mathrm{~s}, t_{o}+13 \mathrm{~s}, t_{o}+20 \mathrm{~s}$, of one side of a dendrite growing at velocity $V=64 \mu \mathrm{m} / \mathrm{s}$. The length of the region shown here is of the order of $L=2.2 \mathrm{~mm}$.

If both $l_{\mathrm{E}}=\tau V$ and $\bar{\lambda}$ obtained for various dendrites are plotted as a function of the diffusion length $l_{D}^{T}$ at the tip, no simple relation is found. In particular for slow dendrites $l_{\mathrm{E}}$ $=\tau V$ and $\bar{\lambda}$ become very large as compared to $l_{D}^{T}$. This may appear surprising because the independent growth of different dendrites would be expected to occur whenever the dendrites are separated by a distance larger than a few diffusion lengths. But the question of the independent growth of two

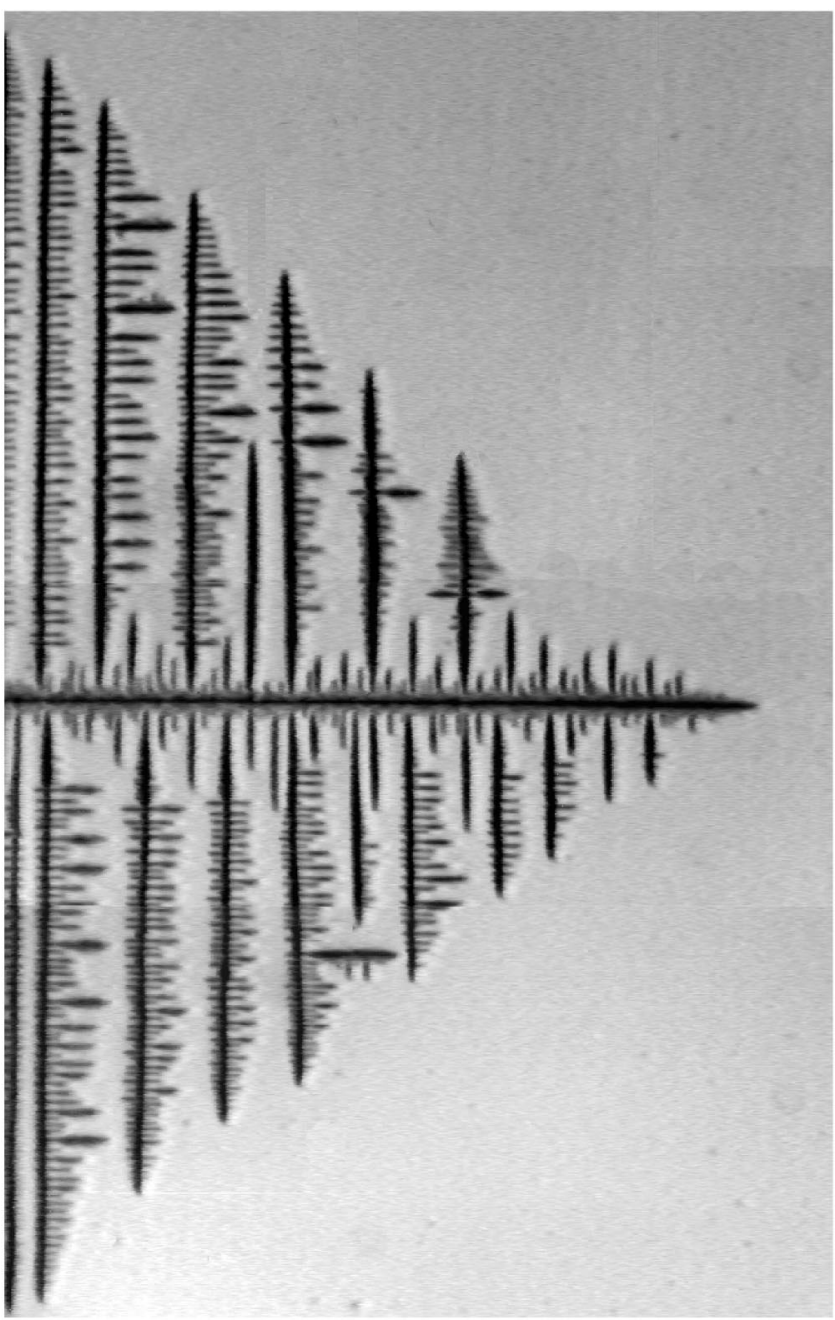

FIG. 12. Photograph of the global structure of a dendrite growing at velocity $V=87 \mu \mathrm{m} / \mathrm{s}$. The length of the region shown here is $L=3.6 \mathrm{~mm}$.

side branches differs from that of the independent growth of parallel dendrites. In order to become free, the branches must first escape out of the diffusion field of the lateral side of the primary dendrite. For very fast dendrites this occurs rather close to the tip in a region where the lateral front is still growing fast and the diffusion length is of the order of $l_{D}^{T}$. In contrast, in slower dendrites, the escape occurs much further away from the tip in a region where the mean motion is slow and where the local diffusion length has thus become large. Only branches much further away from each other can then become independent.

A reasonable assumption is that a branch can only become free when it has become larger than some fraction of the local mean diffusion length of the lateral front. This length at a given abscissa $x$ grows as the square root of time [relation (6)]. Since the growth exponents $\alpha_{n}$ are larger than 0.5 , the branches can, in principle, approach the diffusion front.

For these reasons the relevant comparison is that of both $l_{\mathrm{E}}=\tau V$ and $\bar{\lambda}$ with the local lateral diffusion length $l_{D}^{S}$ in the region where this escape occurs. This length $l_{D}^{S}$ can be computed fixing $t=\tau$ in relation (6) and using the values of $D$ and 


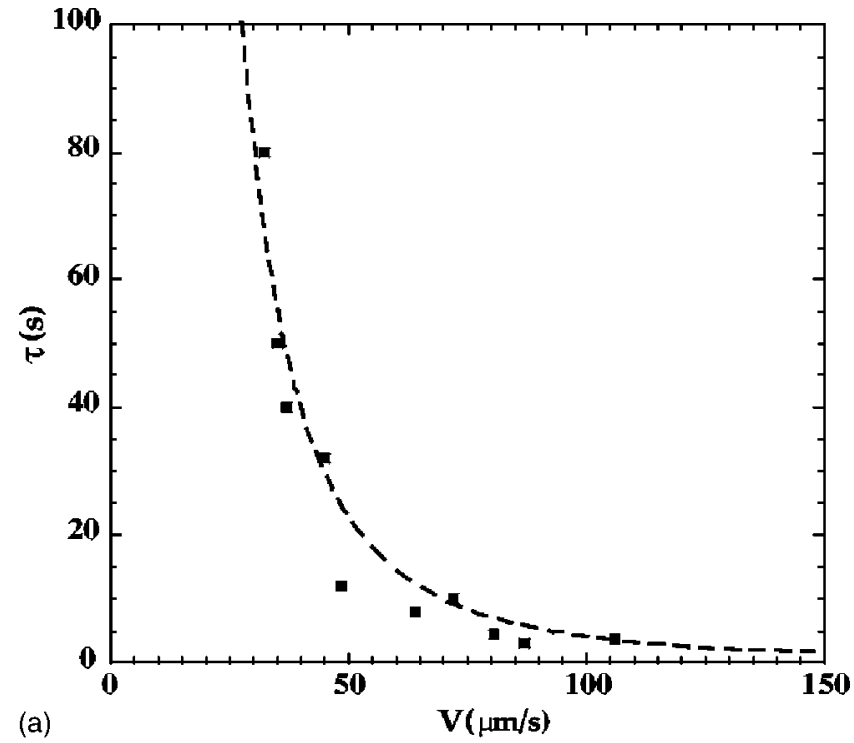

(a)

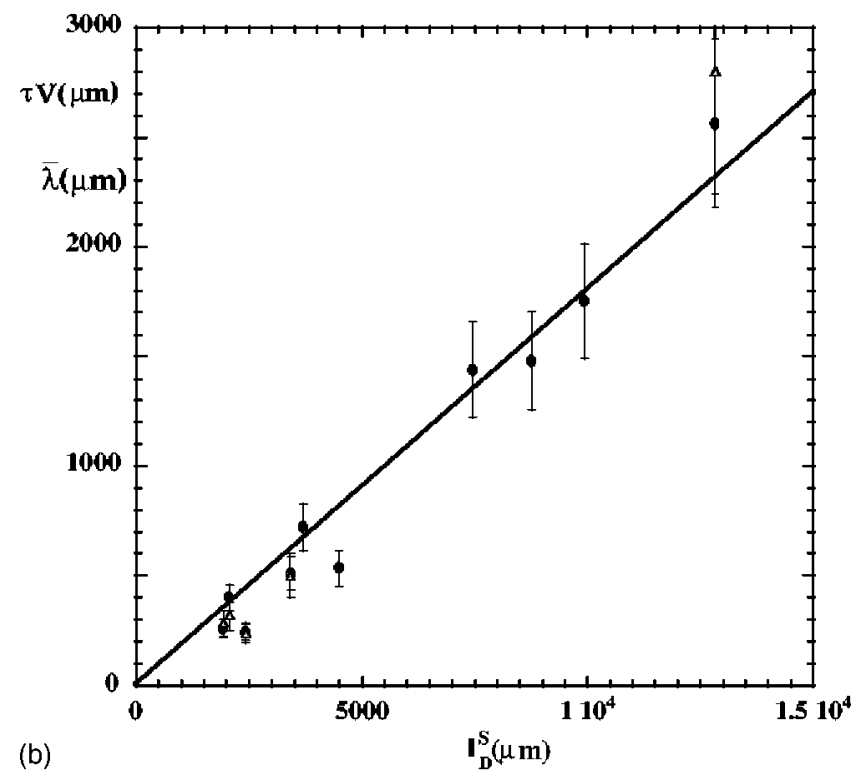

FIG. 13. (a) Evolution of the time of escape $\tau$ as a function of the dendrite's velocity $V$. The curve is the fit by relation (14). (b) Plot of the length of escape $\tau V$ (black dots) and of $\bar{\lambda}$ the mean distance between free branches (open triangles) as a function of $l_{D}^{S}$ the lateral diffusion length in the region of escape. The line is the fit by relation (13).

$k$ specific to our experiment. The resulting plot of $\tau V$ and $\bar{\lambda}$, as a function of $l_{D}^{S}$, given on Fig. 13(b) shows a linear dependence. In our experimental conditions the best fit is

$$
\tau V \approx(0.18 \pm 0.03) l_{D}^{S}
$$

In other terms, as time passes, a branch can escape out of the mean lateral front when its distance to the tip (or its distance to its neighbors) becomes larger than a fifth of the local diffusion length. It can be noted, however, that the data for very slow dendrites are slightly above the linear fit. This could be due to the scarcity of branches with large $\alpha_{n}$ at these velocities.
The linear relation (13) can be used to find an empirical relation for the time $\tau$ as a function of $V$. In our experimental conditions (i.e., $D=2 \times 10^{-9} \mathrm{~m}^{2} / \mathrm{s}$ and $\rho^{2} V=k=7.5 \mu \mathrm{m}^{3} / \mathrm{s}$ ) we find

$$
\tau \approx\left(4 \times 10^{5}\right) V^{-2.5}
$$

which provides a good fit for the measured values [Fig. 13(a)].

\section{DISCUSSION AND CONCLUSION}

The measurements performed on very slow dendrites have provided a phenomenological description of a process which could be called convective coarsening. Dougherty et $a l .{ }^{17}$ recorded, as a function of time the half width of the dendrite at a constant distance from the tip. Close to the tip the power spectrum corresponds to the oscillations due to the formation of the branches. This spectrum exhibits a broad peak at a temporal frequency $f_{t}$ corresponding to a maximum of the amplification in the tip region. This maximum corresponds to the observed mean wavelength of the side branches which is of the order of $4 \rho$ in this region. However, all the frequencies $f<f_{t}$ are also amplified and there is a shift towards more amplification of the low frequency further away from the tip, as coarsening takes place.

A way to understand this process is to note that the mean normal velocity of the lateral front of a dendrite decreases constantly because of the curvature of the parabola. In order to understand the wavelength selection near the tip, Caroli et al. [16] used this argument to perform a modified version of the Mullins and Sekerka linear stability analysis. They computed the shift of the most amplified wavelength as the disturbance is advected away from the tip. Their analysis was classical with the surface tension being the stabilizing factor of the instability.

Here the coarsening should rather be analyzed in the framework of the mean field theory introduced by Brener et al. $[31,32]$. In this theory the Laplacian itself plays the role of an effective surface tension. It should be noted that the coarsening observed here concerns highly nonlinear spiky branches which are very different from the growth of a sinusoidal disturbance of weak amplitude. However, the general process is the same; as the wave packets are advected away from the tip, the normal velocity decreases and the range of amplified wavelengths shifts to larger and larger values. Constantly decreasing frequencies of the initial noise are thus amplified. This is yet another generalization of the convective character of the instability [8]. From this viewpoint there is a striking analogy of the coarsening with the evolution of the instabilities of a shear flow in the mixing layer configuration [25]. In such experiments the initial destabilization gives rise to irregular vortices with a preferential wavelength. Further downstream, as the shear layer becomes thicker, there is an amplification of lower frequencies and the vortices undergo pairing processes by which the pattern's wavelength grows.

The same process can also be understood as the result of the competition of parallel needles growing in a long-range diffusion field. In this perspective a slightly larger branch 
grows faster than its neighbors by a simple point effect. It does so at the expense of the growth of its neighbors. Since the mean growth is always maintained, the material that would have crystallized on the weaker branches is redistributed and contributes to the growth of faster branches with larger $\alpha_{n}$. As a dominant branch grows, however, it becomes sensitive to the presence of other dominant branches located at larger distances along the lateral front. We have shown that this screening is a function of the sum of tangents of the angles $\theta_{q}^{r}$ and $\theta_{q}^{s}$. It will only have a noticeable effect when the lengths (or rather the length differences) of these branches will become comparable to their spacing. In practice, with branches resulting from the normal noise, the screening effect remains weak between branches separated by a distance larger than their own length.

For faster dendrites, the range of values of the exponents $\alpha_{n}$ corresponding to the normal random fluctuations of the tip, shifts to larger values. This can be understood if we consider the same disturbance of the interface in dendrites of various velocities. The smaller the diffusion length, the larger the number of isoconcentration lines disturbed by a protrusion of a given amplitude. For this intuitive reason the same disturbance will grow with larger $\alpha$ 's in faster dendrites. This is confirmed by the results of the simulations using the phase field model presented in Part II.

For fast dendrites there is a crossover; at a certain distance from the tip, the dominant branches are observed to accelerate before reaching the constant velocity of a free dendrite. The transition to this regime of growth is subcritical: in the intermediate range, the branches resulting from the normal noise at the tip remain prisoner of the thickening lateral diffusion field of the dendrite. Only branches due to a large disturbance of the tip can reach the escape velocity. At very large velocities this escape occurs near the tip so that a wedge shaped array is formed, which occupies a finite fraction of the plane.

\section{ACKNOWLEDGMENTS}

We are grateful to M. Adda Bedia, V. Hakim, and P. Tabeling for useful discussions during the course of this work. We acknowledge financial support of the Dirección General de Investigación (Spain) under Project Nos. BFM2003-07850-C03-02 and BFM2003-07749-C05-04.

\section{APPENDIX}

A phenomenological description of this process can be given in the following way. The largest values of the $\alpha_{n}$ are a function of the dendrite's velocity. In a piecewise linear approximation we find that $\alpha_{n}^{\max } \approx 0.5+0.01 \mathrm{~V}$ for $10<\mathrm{V}$ $<50 \mu \mathrm{m} / \mathrm{s}$, and $\alpha_{n}^{M a x}=1$ for $V \geqslant 50 \mu \mathrm{m} / \mathrm{s}$.

If we write that a branch becomes free when its length equals the local diffusion length of the mean front we get

$$
\sqrt{2 \rho V} t^{0.5+0.01 V}=\left(2 D \sqrt{\frac{2}{\rho V}}\right) t^{0.5} .
$$

We use the selection condition of our dendrite. In our experimental conditions we found $\rho^{2} V=7.5 \mu \mathrm{m}^{3} / \mathrm{s}$.

For $V<50 \mu \mathrm{m} / \mathrm{s}$ the fastest branches catch up the mean diffusion front at a time

$$
\tau_{t}=\left(\frac{2 D}{\sqrt{7.5 V}}\right)^{(1 / 0.01 V)}
$$

For velocities larger than $50 \mu \mathrm{m} / \mathrm{s}$ the fastest branch grow with $\alpha_{n}=1$ so that there is a crossover to a value of this time:

$$
\tau_{t}=\left(\frac{2 D}{\sqrt{7.5 V}}\right)^{2}
$$

These times provide an empirical fit to the observed values of $\tau$.
[1] G. P. Ivantsov, Dokl. Akad. Nauk SSSR 58, 567 (1947).

[2] J. S. Langer, in Chance and Matter, Les Houches Lectures XLVI edited by J. Souletie, J. Vannimenus, and R. Stora (Elsevier, New York, 1987).

[3] D. Kessler, J. Koplik, and H. Levine, Adv. Phys. 37, 255 (1988).

[4] M. Ben Amar, Phys. Rev. A 41, 2080 (1990).

[5] Y. Pomeau and M. Ben Amar, in Solids Far from Equilibrium, edited by C. Godrèche (Cambridge University Press, Cambridge, England, 1992).

[6] M. Ben Amar and E. Brener, Phys. Rev. Lett. 71, 589 (1993).

[7] Y. Couder, in Perspectives in Fluid Dynamics, edited by G. K. Batchelor, H. K. Moffat, and M. G. Vorster (Cambridge University Press, Cambridge, England, 2000).

[8] M. Rabaud, Y. Couder, and N. Gerard, Phys. Rev. A 37, 935 (1988).

[9] P. Meakin, Phys. Rev. A 36, 332 (1987).

[10] J. P. Eckmann, P. Meakin, I. Procaccia, and R. Zeitak, Phys. Rev. A 39, 3185 (1989).
[11] S. C. Huang and M. E. Glicksman, Acta Metall. 29, 701 (1981).

[12] R. I. Trivedi, Metall. Trans. A 15, 977 (1984).

[13] R. Pieters and J. S. Langer, Phys. Rev. Lett. 56, 1948 (1986).

[14] J. S. Langer, Phys. Rev. A 36, 3350 (1987).

[15] M. N. Barber, A. Barbieri, and J. S. Langer, Phys. Rev. A 36, 3340 (1987).

[16] B. Caroli, C. Caroli, and B. Roulet, J. Phys. (France), 48, 1423 (1987).

[17] A. Dougherty, P. D. Kaplan, and J. P. Gollub, Phys. Rev. Lett. 58, 1652 (1987).

[18] A. Dougherty and J. P. Gollub, Phys. Rev. A 38, 3043 (1988).

[19] Y. Couder, F. Argoul, A. Arnéodo, J. Maurer, and M. Rabaud, Phys. Rev. A 42, 3499 (1990).

[20] A. Dougherty and R. Chen, Phys. Rev. A 46, R4508 (1992).

[21] E. Brener and D. Temkin, Phys. Rev. E 51, 351 (1995).

[22] Q. Li and C. Beckermann, Acta Mater. 47, 2345 (1999).

[23] D. Kessler and H. Levine, Europhys. Lett. 4, 215 (1987).

[24] Y. Couder, in Branching in Nature, edited by V. Fleury, J. F. 
Gouyet, and M. Leonetti (Springer Verlag, Berlin, 2001), pp. $1-22$.

[25] P. Huerre and P. A. Monkewitz, J. Fluid Mech. 159, 151 (1985).

[26] P. Bouissou, A. Chiffaudel, B. Perrin, and P. Tabeling, Europhys. Lett. 13, 89 (1990).

[27] X. W. Qian and H. Z. Cummins, Phys. Rev. Lett. 64, 3038 (1990).

[28] J. Maurer, B. Perrin, and P. Tabeling, Europhys. Lett. 14, 575
(1991).

[29] V. Hakim, in Scale Invariance, Interfaces and Non-equilibrium Dynamics, edited by A. McKane, M. Droz, J. Vannimenus, and D. Wolf (Plenum Press, New York, 1995), pp. 63-83.

[30] M. Adda Bedia, Ph.D. thesis, Université Paris VI, 1994.

[31] V. Hakim (private communication).

[32] E. Brener, H. Levine, and Yuhai Tu, Phys. Rev. Lett. 66, 1978 (1991). 